\section{Overview of the optical properties of fluorescent nanoparticles for optical imaging}

\author{
Federico Boschi, ${ }^{1}$ Francesco De Sanctis ${ }^{2}$ \\ ${ }^{1}$ Department of Computer Science, \\ University of Verona \\ ${ }^{2}$ Department of Medicine, Immunology \\ Section, Policlinico G.B. Rossi, Verona, \\ Italy
}

\begin{abstract}
Nanoparticles (NPs) include a wide group of small structures composed by very different materials and characterized by peculiar properties that make them suitable for many applications, especially imaging and drug delivery. In this overview, we focus on the optical properties of fluorescent NPs available for in vivo, in vitro and ex vivo preclinical studies and detectable with the optical imaging technique alone or in combination with microscopic confocal imaging. We summarize here the basic principles of the optical detection of fluorescent NPs, elucidating which are the current issues to be resolved and possible solutions to achieve the highest sensitivity and specificity for an unbiased analysis. So far NPs application in clinic is in evaluation due to safety questions still unaddressed but in the future they could dramatically improve both preclinical research and patient clinical care.
\end{abstract}

\section{Introduction}

In the molecular imaging landscape a wide variety of nanoparticles (NPs) with many different physical chemical and pharmacological features has recently appeared. ${ }^{1,2}$ NPs are structures with all the three dimensions sized in the range of 1-100 nanometers, made of metals, semiconductors or polymers, with peculiar optical, magnetic, electronic and structural properties making them suitable for several in vitro and in vivo applications. They are designed especially for diagnostic imaging purposes or as drug-carriers for therapy studies, ${ }^{3,4}$ or for both diagnosis and therapy opportunities in a single theranostic agent. ${ }^{5}$ Their implementation in clinic is currently under evaluation. ${ }^{6}$ A summary of NPs' properties is beyond of the aim of this overview; here we will focus instead on the fluores- cent properties of the NPs synthesized for optical imaging (OI) applications (i.e., for in vivo deep tissue analysis) with a glance on the confocal microscopic imaging field for a more detailed NPs localization in the tissue at the cellular level..$^{7-9}$

The term OI is sometimes used to indicate a large variety of imaging techniques based on the detection of optical photons but more often it designates a specific technique, as we do throughout this overview, developed for in vivo acquisition of optical photons coming from small laboratory animals or, in general, from few centimeters sized-samples. The great improvements of $\mathrm{OI}$ in the last years are due to the high sensitivity achieved by optical detectors and to the high efficiency of the optical probes and light sources, especially in the near infrared region (NIR) of the light spectrum. In fact, OI reveals photons in the ultraviolet (UV, $10-400 \mathrm{~nm})$, visible (400-700 $\mathrm{nm})$ and NIR (700 nm - $1 \mathrm{~mm}$ ) range, but only NIR photons travel through biological tissues with minimal absorption, making them able to reach deeper tissue levels.

\section{Photon absorption}

When photons pass through a biological tissue, they can be absorbed by molecules they interact with. The absorption is modeled by the Lambert-Beer law which describes how the number of photons in a light beam is reduced in an exponential way increasing the thickness of tissue slab. ${ }^{10}$ The distance travelled by photons, represented by the "mean free path before absorption, or absorption length" is in a strict dependence on the tissue optical properties and the light wavelength. The absorption length can be calculated by means of the Lambert-Beer law as the depth at which the number of photons in the beam is reduced to $1 / \mathrm{e}(\mathrm{e}=$ 2.718 ), i.e. around $30 \%$ of the initial value.

Hemoglobin (both in oxidized and reduced form), water, lipids and melanin are the molecules with highest ability to absorb photons of the optical light. ${ }^{11}$ The biological tissues, instead, are optically thin in the 650-800 nm interval, called "NIR optical window", allowing photons of that spectral region to travel a longer distance before being absorbed. For instance, in the muscle tissue, blue photons ( $450 \mathrm{~nm}$ ) have a mean absorption length of about $0.4 \mathrm{~cm}$, whereas NIR photons $(800 \mathrm{~nm})$ of $1.8 \mathrm{~cm} .^{12}$ The differential photons' absorption is clearly visible when a finger is placed on the white LED of the cellular torch: only red photons are visible on the opposite side of the finger whereas the others are "lost" in the tissue.
Correspondence: Federico Boschi, Department of Computer Science, University of Verona, Strada le Grazie 15, 37134 Verona, Italy.

E-mail: federico.boschi@univr.it

Key words: Nanoparticles; optical imaging; fluorescence.

Received for publication: 18 June 2017

Accepted for publication: 26 July 2017.

This work is licensed under a Creative Commons Attribution-NonCommercial 4.0 International License (CC BY-NC 4.0).

(C) Copyright F. Boschi and F. De Sanctis, 2017 Licensee PAGEPress, Italy

European Journal of Histochemistry 2017; 61:2830 doi:10.4081/ejh.2017.2830

This also explains why OI acquisition in the NIR range is particularly suitable for preclinical studies on small animal models (1.5 $\mathrm{cm}$ thick, in case of mice). On the other hand, for the same reason, OI is only marginally used on humans so far, since most of the organs are deeper than $2 \mathrm{~cm}$ and the optical thickness reduces both the excitation light, travelling from the surface to deeper tissue layers, and the fluorescence emission travelling to the surface, beyond which it could be detected.

A molecule that adsorbs a photon is excited to a higher vibrational energy level and rapidly loses part of the energy as heat. It eventually relaxes to the ground state with the emission of lower energy photon. This process, called "fluorescence", is almost instantaneous $\left(10^{-8} \mathrm{~s}\right)$, thus fluorescence emission immediately ends after switching off the excitation source. ${ }^{10}$ When absorption/emission occurs in fluorochromes naturally present in biological structures, the emission is called "autofluorescence" and mostly limits the sensitivity of fluorescent imaging. Fluorescent dyes loaded on or linked to NPs exploit the same physical process to convert excitation in emission light. The fluorescence of semiconductor NPs, like quantum dots (QDs), is due to the same process which involves the valence and the conduction bands instead of single energy levels.

\section{Photon scattering}

Before absorption, the critical processes encountered by the light interacting with the tissues can be photon reflection, refraction, diffusion or scattering, which affect the straight trajectory of the light inside a tis- 
sue. ${ }^{13}$ In particular, the elastic scattering occurs when change of directions do not cause energy losses (i.e., frequency). ${ }^{14}$ Moreover, a photon can be subjected to multiple scattering events (10-1000) before experiencing a fatal encounter with a molecule and disappearing from the light beam. Scattering events are responsible for the "lack of memory" of the initial motion direction of the photons. This is the reason why, putting again a finger on the white LED of the cellular torch, no sign of the finger's bone can be observed. Contrary to $\mathrm{x}$-ray imaging, in which high energy photons travel in straight direction inside the soft tissues and are absorbed by bones, optical photons travel almost randomly in the flesh around the bone from the light source to the opposite surface and exit with many different directions; thus the light emission appears homogenous. ${ }^{15}$

\section{OI instrument}

Specifically designed instruments for OI, named "optical imagers", are usually composed by a tight light box to prevent contamination in the measurement from the ambient light and a charge coupled device (CCD) camera, which is a very sensitive detector composed by 512 x 512 up to 2048 x 2048 pixels. To reduce the electronic noise the CCD is cooled between -40 and $90^{\circ} \mathrm{C}$. The instruments are supplied with a set of excitation and emission filters which allow to choose specific excitation and emission wavelengths respectively, in accordance with the fluorescent dye properties. As light source, a xenon lamp or different lasers (for example 635, 670, 745 and $790 \mathrm{~nm}$ in the FMT instrument, Perkin Elmer, Waltham, MA, USA) are often employed. Mice are placed under anesthesia inside the instrument on a heated stage $\left(37^{\circ} \mathrm{C}\right)$ to preserve the body temperature during acquisitions. A field of view (FoV) from few to $20-30 \mathrm{~cm}$ allows imaging of more than one, and up to five mice at the same time. Diaphragm, binning (i.e., the sensitivity of the CCD), exposure time and focus height can also be selected by the operator. At the end of each acquisition, luminescent images are shown in pseudocolors that are associated to the intensity of the light reaching each pixel and overlapped with a picture of the animals to localize the regions of light signal source.

The quantification of fluorescence emission is performed by tracing a region of interest (ROI) on the luminescent images and by calculating the light efficiency that is obtained normalizing the fluorescent emis- sion with the incident excitation intensities. This measurement is not influenced by the camera settings. The acquisition time used generally for fluorescent imaging (FLI) is in the order of seconds but it can go up to 10 $\min$ for $3 \mathrm{D}$ acquisitions, since they require multiple exposures.

Apart from fluorescence, OI is able to detect also bioluminescence sources (bioluminescence imaging, BLI). The most representative application is the light production derived from the enzymatic reaction of luciferin oxidation catalyzed by luciferase enzyme for in vivo monitoring of tumor growth or for in vivo ${ }^{16,17}$ and in vitro reporter studies. ${ }^{18}$ In both scenarios, immortalized cells or mice used in these studies need to be genetically engineered with luciferase gene since they do not physiologically produce this protein. Recently, other optical imaging-based applications were developed exploiting the registration of the Cerenkov luminescence (CLI) ${ }^{19-21}$ and of the radioluminescence (RLI) both produced by the interaction of radionuclides with matters. ${ }^{22-24}$ Moreover, NPs used to shift the blue glow of Cerenkov radiation have also been investigated. ${ }^{25,26}$

\section{Fluorescent NPs}

In biological and preclinical studies, fluorescent NPs are generally used to localize molecules or highlight processes in cultured cells or living organisms. In the latter, it is noteworthy that both excitation and emission lights are subjected to absorption and scattering; therefore, both excitation and emission wavelengths should be in the NIR optical window to allow good signal detection. Consequently, best OI-optimized florescent NPs have 700-750 nm excitation and 750-800 $\mathrm{nm}$ emission wavelengths, respectively. ${ }^{27}$ The CCD cameras mounted on OI instruments are generally optimized for visible photon acquisitions but are still sensitive (even if with reduced sensitivity) in the NIR range, thus they can detect photons in the $800-850 \mathrm{~nm}$ wavelength range. Working with high efficient NPs is mandatory in order to obtain a good signal to noise ratio and to achieve low exposure time acquisitions; if the efficiency of the NPs is too low, the specific signal of the particles can be covered by autofluorescence of the tissues, ${ }^{28}$ especially of the skin. ${ }^{29}$ Thus, nonetheless NIR-developed dyes are more suitable for deep-tissue imaging, autofluorescence can still limit detection sensitivity.

$\mathrm{OI}$ is a very versatile, fast and non-invasive technology but have some limitations: first of all, OI alone is not sufficient to accu- rately define NPs localization. In other words, most of OI instruments provide 2D images without any information of the sources' depth. New generation instruments and 3D modeling software ${ }^{30,31}$ tried to fill this gap. However, 3D reconstructions of fluorescent images require longer exposure times and the results are affected by the hypothesis on which the software is based. Secondly, the scattering process creates a spot of light on the imaged surface also in the case of perfectly point sources inside the tissues. Finally, photon absorption can lead to a misunderstanding of the light signal distribution. For example, lack of light signal in a specific anatomic region could be interpreted as absence of NPs instead of high photon tissue absorption. Consequently, also the shape of target organs could be altered by the absorption of different tissue layers interposed between the emission sources and the detector. ${ }^{15}$ However, lower resolution and signal broadening are rewarded by very high sensitivity, aiming to single fluorescently labelled cell detection, at least for in vitro acquisitions. For example, for dual contrast agents (MRI - OI) only one fluorophore is needed for optical detection against 100 molecules with magnetic properties for MRI visualization.

To improve NPs tissue localization with OI, an acquisition of the dissected organs is often suggested. Unfortunately, the time lapse necessary for ex vivo acquisitions critically affects the morphological architecture of the tissue (especially for those requiring immediate fixation, such as pancreas) making them almost useless for further microscopic investigations. Moreover, an untreated control group, which does not receive NPs, is always necessary to establish organs' autofluorescence emission in order to evaluate statistically significant differences with the treated group. Finally, perfusion of the animals is required to remove NPs in the blood vessels and to acquire fluorescence emission from NPs in the parenchima. In conclusion, transmission electron microscopy (TEM) or optical microscopic investigations are still mandatory to perform an unbiased analysis. The second ones are conducted on very thin tissue sections (few $\mu \mathrm{m}$ ) so light absorption and scattering issues are highly reduced. However, the light emitted by NPs, is captured by photomultiplier (PMT) detectors optimized for the acquisition of UV-visible photons and which are almost blind to NIR wavelengths. One possible solution is to use or synthesize NPs with "bluer" emission respect to the "optimum", i.e. around 700 $\mathrm{nm}$ that is still detectable by PMTs. This 
requires an excitation wavelength at least at the beginning of the NIR window. This approach represents a compromise to detect NPs both in vivo (OI) and ex vivo (confocal imaging).

Another approach to obtain NPs with optical properties suitable for both OI and microscopic imaging is to synthesize them with two different fluorophores. ${ }^{32}$ Avoiding cross-contamination of the light signal and unwanted chemical reactions between the two fluorophores, one of them must be chosen in order to obtain excitation/emission of $750 / 800 \mathrm{~nm}$ for OI acquisitions. Instead, for confocal imaging the requirements are less compelling. Many laser lines can be used for confocal microscopy $(405,458,475$, $488,495,514,543$, and $633 \mathrm{~nm}$ in a diffuse TCS SP5 Leica system) and the range of PMTs sensitivity can be set freely. An example is the NP synthesis with cyanine (Cy) and rhodamine in the silica matrix. The first one $(830 \mathrm{~nm} / 855 \mathrm{~nm})$ is optimized for OI, the second one $(570 \mathrm{~nm} / 587$ $\mathrm{nm})$ for confocal imaging. ${ }^{32}$

\section{Organic fluorophores and quan- tum dots}

In the optical imaging field, many kind of fluorophores can be enrolled. They are mainly classified as molecular systems with a defined structure which include organic and biologically-derived fluorophores, nanocrystal such as QDs and nanometer- to micrometer-sized particles with size independent optical features. ${ }^{33}$ For biomedical imaging applications, the most useful nanosized fluorescent dyes are organic fluorophores conjugated or inserted in NPs structures and QDs. A comparison between organic fluorophores and QDs can be found in literature. ${ }^{34}$

Organic fluorophores can be synthesized with many different emission wavelengths but they show a poor difference between excitation and emission peaks (Stokes shift) up to $50 \mathrm{~nm}$. Low photostability (stability against chemical reactions induced by the incoming radiation) and high photobleaching (photochemical alteration of a dye or a molecule which permanently blocks its ability to fluoresce) generally represent other issues for their applications. Keeping in mind the optical requirements for in vivo applications, Cy5.5 (675 nm / $694 \mathrm{~nm})$ Cy7 (743 nm / $767 \mathrm{~nm})$, Indocyanine green (ICG, $788 \mathrm{~nm} / 813 \mathrm{~nm}$ ) and Alexa Fluor $750(752 \mathrm{~nm} / 776 \mathrm{~nm})$ are good choices.

On the other hand, QDs (1-6 nm core diameter) are very bright fluorophores made from nanocrystals of semiconductor material ${ }^{35,36}$ with peculiar optical properties and advantages: efficiency 20-30 times higher than organic fluorophores, size-tunable emission, extremely broad excitation range and narrow emission which allow large Stokes shift (resulting in an increase of the signal/noise ratio in case of tissue autofluorescence). High photostability and low photobleaching are their strength, often assured by an outer shell which protects the NP core. Unfortunately, QDs toxicity, especially of those containing cadmium (i.e., CdSe and CdTe), limits their use in vivo and leads to the development of new kind of QDs with less harmful chemical elements, such as InP/InGaP QDs. For OI applications, QDs with emission at 750-800 nm range are the best choice.

The blinking, or fluorescence intermittency, is the switching between $\mathrm{ON}$ and OFF (bright and dark) states of the emitters under continuous excitation, with the latter being a property of the nanoscale fluorophores (both molecular fluorophores and QDs). ${ }^{37,38}$ For in vivo acquisitions, where only macroscopic effects are detectable (due to the relatively long exposure time and the stochastic nature of the light propagation), it results in a reduction of the fluorescent light signal.

\section{Conclusions}

Optical imaging is a noninvasive technology that exploits physical properties of molecules to highlight specific biological processes. Nonetheless the limitations of both OI technique (tissue adsorption, scattering) and dyes (photobleaching, photostability, blinking) the main concern is represented by NPs' toxicity. Most of them contain elements with carcinogenic, immunotoxic action, such as cadmium and selenium based QDs. Moreover, concentration, size, outer coating bioactivity, charge, and oxidative, mechanical and photolytic stability also play a role in determining NPs' toxicity. ${ }^{39}$ Thus, a great effort is currently being spent to fill this requirement. However, advances in optical instrumentation and new contrast agents allowed to enhance sensitivity and specificity for example of cancer cell detection, providing significant help to surgeon in visualizing cancer margins during tumor resections. NPs' functionalization with ligands targeting cellular surface proteins ${ }^{40-42}$ or cellular metabolic biomarkers $(\mathrm{pH}$, oxidative stress or activation of peculiar proteases), ${ }^{43}$ increased as well sensitivity and specificity of detection in preclinical settings. In the last years, theranostic application of NPs were also investigated in preclinical settings providing encouraging results. ${ }^{44,45}$ These studies will be soon translated in new diagnostic tools to detect the pathology at the earliest stages, therefore increasing the therapeutic intervention window and patient clinical outcome.

\section{References}

1. Padmanabhan P, Kumar A, Kumar S, Chaudhary RK, Gulyas B. Nanoparticles in practice for molecularimaging applications: An overview. Acta Biomater 2016;41:1-16.

2. Berezin MY. Historical Perspective on Nanoparticles in Imaging from 1895 to 2000. Nanotechnology for biomedical imaging and diagnostics. John Wiley \& Sons, Inc.: 2014. p. 1-23.

3. Liu Y, Miyoshi H, Nakamura M. Nanomedicine for drug delivery and imaging: a promising avenue for cancer therapy and diagnosis using targeted functional nanoparticles. Int $\mathrm{J}$ Cancer 2007; 120:2527-37.

4. Chow EK, Ho D. Cancer nanomedicine: from drug delivery to imaging. Sci Transl Med 2013;5:216rv4.

5. Xie J, Lee S, Chen X. Nanoparticlebased theranostic agents. Adv Drug Deliv Rev 2010;62:1064-79.

6. Anselmo AC, Mitragotri S. Nanoparticles in the clinic. Bioeng Transl Med 2016;1:10-29.

7. Costanzo M, Carton F, Marengo A, Berlier G, Stella B, Arpicco S, et al. Fluorescence and electron microscopy to visualize the intracellular fate of nanoparticles for drug delivery. Eur J Histochem 2016;60:2640.

8. Malatesta M. Transmission electron microscopy for nanomedicine: novel applications for long-established techniques. Eur J Histochem 2016;60:2751.

9. Busato A, Fumene Feruglio P, Parnigotto PP, Marzola P, Sbarbati A. In vivo imaging techniques: a new era for histochemical analysis. Eur J Histochem 2016;60:2725.

10. Tuchin V, Engineers SoP-oI. Tissue optics: Light scattering methods and instruments for medical diagnosis. SPIE Press: 2015.

11. Wilson RH, Nadeau KP, Jaworski FB, Tromberg BJ, Durkin AJ. Review of short-wave infrared spectroscopy and imaging methods for biological tissue characterization. J Biomed Opt 2015; 20:030901. 
12. Jacques SL. Optical properties of biological tissues: a review. Phys Med Biol 2013;58):R37-61.

13. Council NR. Mathematics and physics of emerging biomedical imaging. Washington, DC: National Academies Press: 1996.

14. Brancewicz M, Itou M, Sakurai Y. A Monte Carlo study of high-energy photon transport in matter: application for multiple scattering investigation in Compton spectroscopy. J. Synchrotron $\operatorname{Rad} 2016 ; 23: 244-52$

15. Hillman EM, Amoozegar CB, Wang T, McCaslin AF, Bouchard MB, Mansfield $\mathrm{J}$, et al. In vivo optical imaging and dynamic contrast methods for biomedical research. Philos Trans A Math Phys Eng Sci 2011;369:4620-43.

16. Luker KE, Luker GD. Bioluminescence imaging of reporter mice for studies of infection and inflammation. Antiviral Res 2010;86:93-100.

17. Stellari F, Bergamini G, Ruscitti F, Sandri A, Ravanetti F, Donofrio G, et al. In vivo monitoring of lung inflammation in CFTR-deficient mice. J Transl Med 2016;14:226.

18. Prescher JA, Contag CH. Guided by the light: visualizing biomolecular processes in living animals with bioluminescence. Curr Opin Chem Biol 2010;14:80-9.

19. Spinelli AE, Boschi F. Novel biomedical applications of Cerenkov radiation and radioluminescence imaging. Phys Med 2015;31:120-9.

20. Spinelli AE, Schiariti MP, Grana CM, Ferrari M, Cremonesi M, Boschi F. Cerenkov and radioluminescence imaging of brain tumor specimens during neurosurgery. J Biomed Opt 2016;21: 50502.

21. Spinelli AE, Ferdeghini M, Cavedon C, Zivelonghi E, Calandrino R, Fenzi A, et al. First human Cerenkography. J Biomed Opt 2013;18:20502.

22. Spinelli AE, Lo Meo S, Calandrino R, Sbarbati A, Boschi F. Optical imaging of Tc-99m-based tracers: in vitro and in vivo results. J Biomed Opt 2011;16: 116023.

23. Boschi F, Meo SL, Rossi PL, Calandrino R, Sbarbati A, Spinelli AE. Optical imaging of alpha emitters: simulations, phantom, and in vivo results. $\mathrm{J}$ Biomed Opt 2011;16:126011.
24. Boschi F, Pagliazzi M, Rossi B, Cecchini MP, Gorgoni G, Salgarello M, et al. Small-animal radionuclide luminescence imaging of thyroid and salivary glands with Tc99m-pertechnetate. J Biomed Opt 2013;18:76005.

25. Shaffer TM, Pratt EC, Grimm J. Utilizing the power of Cerenkov light with nanotechnology. Nat Nanotechnol 2017;12:106-17.

26. Boschi F, Spinelli AE. Quantum dots excitation using pure beta minus radioisotopes emitting Cerenkov radiation. Rsc Adv 2012;2:11049-52.

27. Ravoori MK, Singh S, Bhavane R, Sood AK, Anvari B, Bankson J, et al. Multimodal magnetic resonance and near-infrared-fluorescent imaging of intraperitoneal ovarian cancer using a dual-mode-dual-gadolinium liposomal contrast agent. Sci Rep 2016;6:38991.

28. Bouchard MB, MacLaurin SA, Dwyer PJ, Mansfield J, Levenson R, Krucker $\mathrm{T}$. Technical considerations in longitudinal multispectral small animal molecular imaging. J Biomed Opt 2007;12: 051601.

29. Mansfield JR, Gossage KW, Hoyt CC, Levenson RM. Autofluorescence removal, multiplexing, and automated analysis methods for in-vivo fluorescence imaging. J Biomed Opt 2005;10: 41207.

30. Kuo C, Coquoz O, Troy TL, Xu H, Rice BW. Three-dimensional reconstruction of in vivo bioluminescent sources based on multispectral imaging. J Biomed Opt 2007;12:024007.

31. Srinivasan S, Ghadyani HR, Pogue BW, Paulsen KD. A coupled finite elementboundary element method for modeling Diffusion equation in 3D multi-modality optical imaging. Biomed Opt Express 2010;1:398-413.

32. Rampazzo E, Boschi F, Bonacchi S, Juris R, Montalti M, Zaccheroni N, et al. Multicolor core/shell silica nanoparticles for in vivo and ex vivo imaging. Nanoscale 2012;4:824-30.

33. Seydack M. Nanoparticle labels in immunosensing using optical detection methods. Biosens Bioelectron 2005;20: 2454-69.

34. Resch-Genger U, Grabolle M, Cavaliere-Jaricot S, Nitschke R, Nann T. Quantum dots versus organic dyes as fluorescent labels. Nat Methods 2008;5:
763-75.

35. Alivisatos AP. Semiconductor clusters, nanocrystals, and quantum dots. Science 1996;271:933-7.

36. Weller H. Quantum size colloids: From size-dependent properties of discrete particles to self-organized superstructures. Curr Opin Colloid Interface Sci 1998;3:194-9.

37. Stefani FD, Hoogenboom JP, Barkai E. Beyond quantum jumps: Blinking nanoscale light emitters. Phys Today 2009;62:34-9.

38. Frantsuzov P, Kuno M, Janko B, Marcus RA. Universal emission intermittency in quantum dots, nanorods and nanowires. Nat Phys 2008;4:519-22.

39. Stern ST, McNeil SE. Nanotechnology safety concerns revisited. Toxicol Sci 2008;101:4-21.

40. Zhang X, Bloch S, Akers W, Achilefu S. Near-infrared molecular probes for in vivo imaging. Curr Protoc Cytom 2012; Chapter 12:Unit 12 27. doi: 10.1002/0471142956.cy1227s60.

41. Funovics M, Montet X, Reynolds F, Weissleder R, Josephson L. Nanoparticles for the optical imaging of tumor E-selectin. Neoplasia 2005;7: 904-11.

42. Yang L, Mao H, Cao Z, Wang YA, Peng $\mathrm{X}$, Wang X, et al. Molecular imaging of pancreatic cancer in an animal model using targeted multifunctional nanoparticles. Gastroenterol 2009;136:1514-25 e2.

43. Bremer C, Ntziachristos V, Weitkamp B, Theilmeier G, Heindel W, Weissleder R. Optical imaging of spontaneous breast tumors using protease sensing 'smart' optical probes. Invest Radiol 2005;40:321-7.

44. Taratula O, Schumann C, Duong T, Taylor KL, Taratula O. Dendrimerencapsulated naphthalocyanine as a single agent-based theranostic nanoplatform for near-infrared fluorescence imaging and combinatorial anticancer phototherapy. Nanoscale 2015;7:3888-902.

45. Taratula O, Schumann C, Naleway MA, Pang AJ, Chon KJ, Taratula O. A multifunctional theranostic platform based on phthalocyanine-loaded dendrimer for image-guided drug delivery and photodynamic therapy. Mol Pharm 2013;10:3946-58. 